\title{
Memória de Reconhecimento: Modelos de Processamento Simples versus Duplo
}

\author{
Antônio Jaeger - Universidade Federal de Minas Gerais, Belo Horizonte, Brasil
}

\begin{abstract}
Resumo
O processo de reconhecimento de memórias consiste em discriminar informações previamente encontradas de informações novas. Duas abordagens teóricas divergentes buscam explicar esse fenômeno. A primeira propõe que o reconhecimento se baseia essencialmente na sensação de familiaridade gerada por cada estímulo. A segunda propõe que além da intensidade da familiaridade, a evocação de aspectos qualitativos e contextuais dos eventos memorizados possui um papel fundamental no processo de reconhecimento. Aspectos teóricos e metodológicos inerentes às duas linhas teóricas são criticamente discutidos no presente artigo, sendo que o resultado dessa discussão se apresenta favorável à segunda perspectiva teórica.

Palavras-chave: memória, memória episódica, reconhecimento
\end{abstract}

\section{Single - Versus Dual-Process Models of Recognition Memory}

\begin{abstract}
Memory recognition consists in discriminating previously encountered information from novel information. Two divergent theoretical approaches pursue to provide a framework for the process of recognition. The first proposes that memory recognition is based essentially on a sense of familiarity elicited by each stimulus. The second proposes that, in addition to familiarity, the retrieval of qualitative and contextual information inherent to encoded events has an important role during recognition. Theoretical and methodological aspects from both approaches are discussed and the result of this discussion is in favor of the second theoretical approach.

Keywords: memory, episodic memory, recognition
\end{abstract}

Memoria de Reconocimiento: Modelos de Procesamiento Simples o Dobles

\begin{abstract}
Resumen
El proceso de reconocimiento de memorias, consiste básicamente en discriminar informaciones, previamente encontradas en nuevas informaciones. Dos abordajes teóricos divergentes tratan de explicar este fenómeno. El primero sugiere que el reconocimiento de memorias se basa esencialmente en la sensación de familiaridad generada por cada estímulo. El segundo propone que además de la intensidad familiar, la evocación de aspectos cualitativos y contextuales del evento memorizado posee un papel fundamental en el proceso de reconocimiento. Aspectos teóricos y metodológicos inherentes a las dos líneas teóricas, son críticamente discutidos en el presente artículo y el resultado de esta discusión se presenta favorable al segundo abordaje teórico. Palabras-clave: memoria, memoria episódica, reconocimiento
\end{abstract}

Memória episódica é um tipo de memória que envolve o armazenamento de informações referentes a eventos específicos situados no tempo e no espaço (Tulving, 1983). Isto é, consiste no registro de eventos ocorridos ao longo da vida de um indivíduo e que podem ser situados em momentos específicos (i.e., datas, ocasiões) vivenciados por ele. De acordo com Endel Tulving, autor que forneceu as bases para o estudo da memória episódica, ela pode ser caracterizada metaforicamente como uma "viajem no tempo" e necessariamente envolve o processo denominado "consciência autonoética", que consiste na sensação de que o evento lembrado ocorreu da maneira que está sendo recordado e foi vivenciado em primeira pessoa pelo indivíduo que o memorizou (Tulving \& Thomson, 1973).

Uma abordagem experimental muito utilizada para a investigação da memória episódica consiste na utilização de tarefas de memória de reconhecimento
(Yonelinas, 2002). Nesse tipo de tarefa, um conjunto de estímulos é inicialmente apresentado aos participantes de pesquisa (e.g., palavras, figuras, etc.). Após essa apresentação inicial, denominada "fase de codificação", os mesmos estímulos são novamente apresentados entremeados de estímulos que não foram previamente apresentados. Nesse momento, denominado "fase de teste", os participantes identificam quais estímulos foram encontrados na fase de codificação (estímulos "antigos") e quais estímulos estão sendo vistos pela primeira vez (estímulos "novos"). O desempenho nessa tarefa é medido por meio de o quanto os participantes são capazes de discriminar estímulos antigos de estímulos novos.

Os testes de memória de reconhecimento se diferenciam de outros tipos de testes de memória por apresentarem diretamente aos participantes de pesquisa as informações previamente codificadas. Em outro tipo de teste de memória, denominado teste de 
recordar livre, é requerido aos participantes que evoquem os estímulos previamente codificados sem a reapresentação deles e sem nenhum tipo de pista que possa auxiliar nessa evocação. Os participantes são instruídos a simplesmente lembrar o máximo possível de itens codificados (e.g., Glanzer \& Cunitz, 1966). Ainda em outro tipo de teste frequentemente utilizado em pesquisas com seres humanos, denominado teste de Recordar com Pistas, os participantes recebem informações durante a fase de teste que podem auxiliar na evocação dos itens codificados, como o fornecimento das primeiras letras de uma série de palavras que foram previamente codificadas, por exemplo (Greene, 1986). Em contraste aos testes de recordar livre e recordar com pistas, testes de reconhecimento apresentam a particularidade de proporcionar a utilização de medidas que levam em conta a discriminação direta entre itens antigos e itens novos, uma vez que itens novos não são usualmente empregados em outros tipos de teste de memória.

Essa possibilidade de contrastar de maneira direta itens antigos e novos gerou uma questão de pesquisa que atualmente é intensamente debatida, que consiste na especificação dos processos cognitivos subjacentes à memória de reconhecimento. No contexto desse debate, alguns autores defendem a posição de que o reconhecimento é baseado única e exclusivamente em um processo denominado "familiaridade" (Verde \& Rotello, 2007). Esse processo pode ser caracterizado como uma simples sensação de que algo foi previamente encontrado, sendo que essa sensação pode ter várias gradações. Isto é, cada item encontrado durante a fase de codificação gera durante a fase de teste uma sensação de familiaridade que pode ser inserida dentro de um continum entre a total ausência de familiaridade até níveis muito altos de familiaridade. Dessa forma, itens que geram uma sensação de familiaridade mais intensa dentro desse continuum são mais frequentemente julgados como antigos, e itens que geram uma sensação de familiaridade de menor intensidade dentro desse continuum são mais frequentemente classificados como novos. Em contraste com esse posicionamento teórico, outros autores defendem que o reconhecimento é frequentemente composto da combinação de dois processos. Isto é, além do processo gradativo de familiaridade, também seria determinante para o reconhecimento a "recordação" de aspectos contextuais e detalhados da fase de codificação (Yonelinas, 1994). Essa recordação se caracterizaria por um processo discreto (i.e., "tudo ou nada") no qual os julgamentos de memória se baseariam na presença ou ausência da evocação de aspectos contextuais associados aos eventos ou informações memorizadas.

Os autores que defendem o reconhecimento enquanto um processo único (Macmillan \& Creelman, 2005; Verde \& Rotello, 2007; Mickes, Wixted, \& Wais, 2007; Mickes, Hwe, Wais, \& Wixted, 2011) utilizam como base teórica a teoria da Detecção de Sinal (Macmillan \& Creelman, 2005; Green \& Swets, 1966). A partir dessa teoria, a familiaridade eliciada por cada item em um teste de memória é caracterizada como um sinal contínuo ou gradativo de memória. Essa caracterização e as implicações dessa teoria para o estudo da memória de reconhecimento serão abordados na seção seguinte. Os autores que, por outro lado, defendem que além da familiaridade, a recordação também possui um papel importante no processo de reconhecimento, utilizam como base teórica a teoria do Processamento Duplo (Mandler, 1980; Jacoby, 1991; Yonelinas, 1994). Essa teoria será apresentada e discutida na terceira seção do artigo.

Em suma, o objetivo do presente estudo é o de discutir criticamente esses dois modelos teóricos do reconhecimento, apontando as limitações e as vantagens deles. O presente estudo objetiva também a elucidação de questões atuais e direções futuras proporcionadas por esses modelos teóricos. Para isso, os dois modelos serão apresentados de maneira detalhada, e devido à alta quantidade de publicações sobre esse tema, somente os artigos mais relevantes e de maior impacto no âmbito de cada uma dessas abordagens teóricas serão discutidos (para uma revisão exaustiva, ver Yonelinas, 2002). A discussão e apresentação específica de cada modelo teórico será seguida por uma discussão de caráter mais crítico quanto à capacidade de cada modelo de fazer predições válidas e que sejam corroboradas por dados empíricos.

\section{Teoria da Deteçãa de Sinal}

A teoria da Detecção de Sinal propõe que quando se entra em contato com uma série de estímulos ou informações, como na fase de codificação em testes de reconhecimento, há uma grande variabilidade na intensidade com a qual os itens são memorizados (Lockhart \& Murdock, 1970). Essa intensidade é normalmente distribuída com a maioria dos itens distribuídos em torno de uma média geral de intensidade para cada tipo de estímulo (ver Figura 1).

Como pode ser visto na Figura 1, o sinal de memória é representado pela seta abaixo das duas 
distribuições, sendo que ela simboliza o aumento gradativo e contínuo da intensidade do sinal de memória. Assim, itens mais para esquerda possuem um sinal mais fraco de memória, itens localizados mais para a direita, um sinal mais forte (Banks, 1970). Como pode ser visto na Figura 1, embora itens novos e antigos sejam normalmente distribuídos, os itens novos tendem a se distribuir para o lado esquerdo (menor intensidade), enquanto itens antigos tendem a se distribuir para $o$ lado direito (maior intensidade) da figura.

Como pode ser observado também na Figura 1, as duas curvas representando itens novos e antigos são cortadas por uma linha vertical, a qual representa o parâmetro denominado "critério". Critério significa a intensidade do sinal de memória a partir da qual o indivíduo classificará o item como antigo ou novo. Assim, todos os itens que estiverem do lado direito do critério serão considerados antigos, e todos os itens que estiverem ao lado esquerdo serão considerados novos. Em outras palavras, todos os itens que eliciarem um sinal de memória com intensidade maior do que a intensidade do critério estabelecido pelo indivíduo serão classificados como antigos, enquanto todos os itens que eliciarem um sinal de memória com intensidade menor do que a intensidade do critério estabelecido pelo indivíduo, serão classificados como novos (Macmillan \& Creelman, 2005; Banks, 1970).
$\mathrm{Na}$ figura 2, é possível visualizar os quatro tipos de resultado possíveis em um teste simples de reconhecimento. Como apresentado na Figura 1, esses resultados podem ser ilustrados dentro do modelo da detecção de sinal, isto é, dentro da distribuição das respostas para cada tipo de item. Quando há mudança no critério estabelecido pelo indivíduo, entretanto, as frequências de acertos, erros, rejeições corretas e alarmes falsos também se modificam. Assim, quando o critério é movido para a direita, a chance de alarmes falsos é diminuída, ao passo que a chance de erros é aumentada (Figura 3A). Quando o critério é movido para a esquerda, aumenta-se a chance de se emitir alarmes falsos, porém a chance de erros é bastante diminuída (Figura 3B).

Além da modificação do critério, outro aspecto desse modelo que altera a frequência dos tipos de resultados de respostas emitidos pelos sujeitos, é a própria capacidade de discriminar entre os dois tipos de estímulo durante o desempenho da tarefa de reconhecimento. Dentro do modelo da detecção de sinal, a capacidade de discriminar entre os estímulos antigos e novos é expressa por meio da distância entre as duas curvas (Green \& Swets, 1966). Isto é, uma distância maior entre as duas curvas, como na Figura3C, indica que há uma maior capacidade do indivíduo em discriminar itens antigos de novos, enquanto uma menor distância (Figura 3D), indica que a capacidade

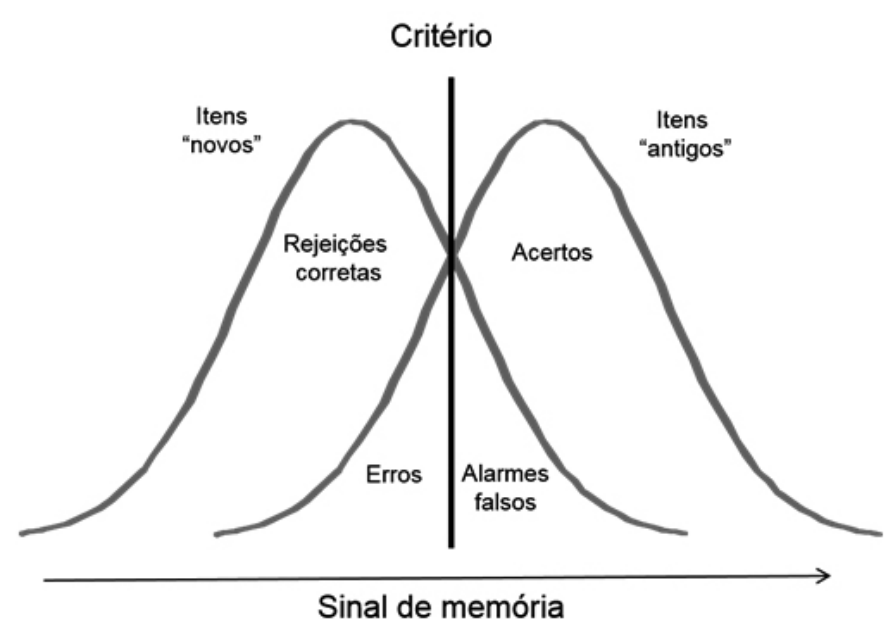

Figura 1. Teoria da detecção de sinal.

Figura 1. Modelo básico da teoria de detecção de sinal, no qual se incluem as distribuições de itens novos e antigos de acordo com a intensidade do sinal de memória eliciado pelos mesmos. Itens novos tendem a se agrupar mais para o lado esquerdo, enquanto itens antigos para o lado direito, indicando que itens antigos eliciam um sinal de memória de forma geral mais intenso do que itens novos. Critério é representado pela linha vertical e os tipos de respostas (acertos, erros, rejeições corretas e alarmes falsos) são representados diretamente nas curvas. 


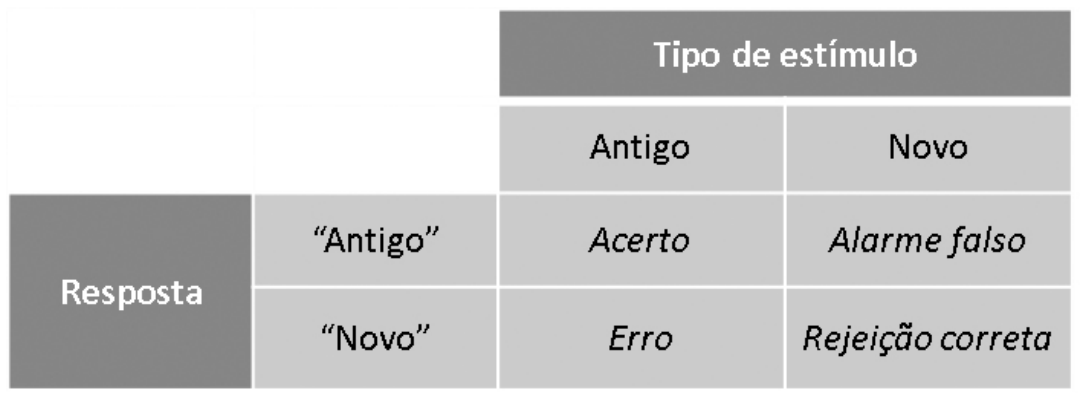

Figura 2. Combinações entre Tipos de Item (antigo ou novo) e Tipos de Resposta (“Antigo" ou "Novo”).

Figura 2. Existem quatro resultados possíveis para cada tentativa de resposta. Dois resultados corretos e dois incorretos. No que tange os corretos, quando o estímulo Antigo é classificado pelo sujeito como "Antigo", têm-se um tipo de resposta denominado "Acerto". Quando o estímulo é Novo e é classificado pelo sujeito como "Novo", têm-se uma "Rejeição correta". No que se refere aos possíveis resultados incorretos para cada tentativa, o tipo de estímulo Antigo classificado pelo sujeito como "Novo" gera um "Erro", enquanto um estímulo Novo classificado como "Antigo" gera um "Alarme falso".
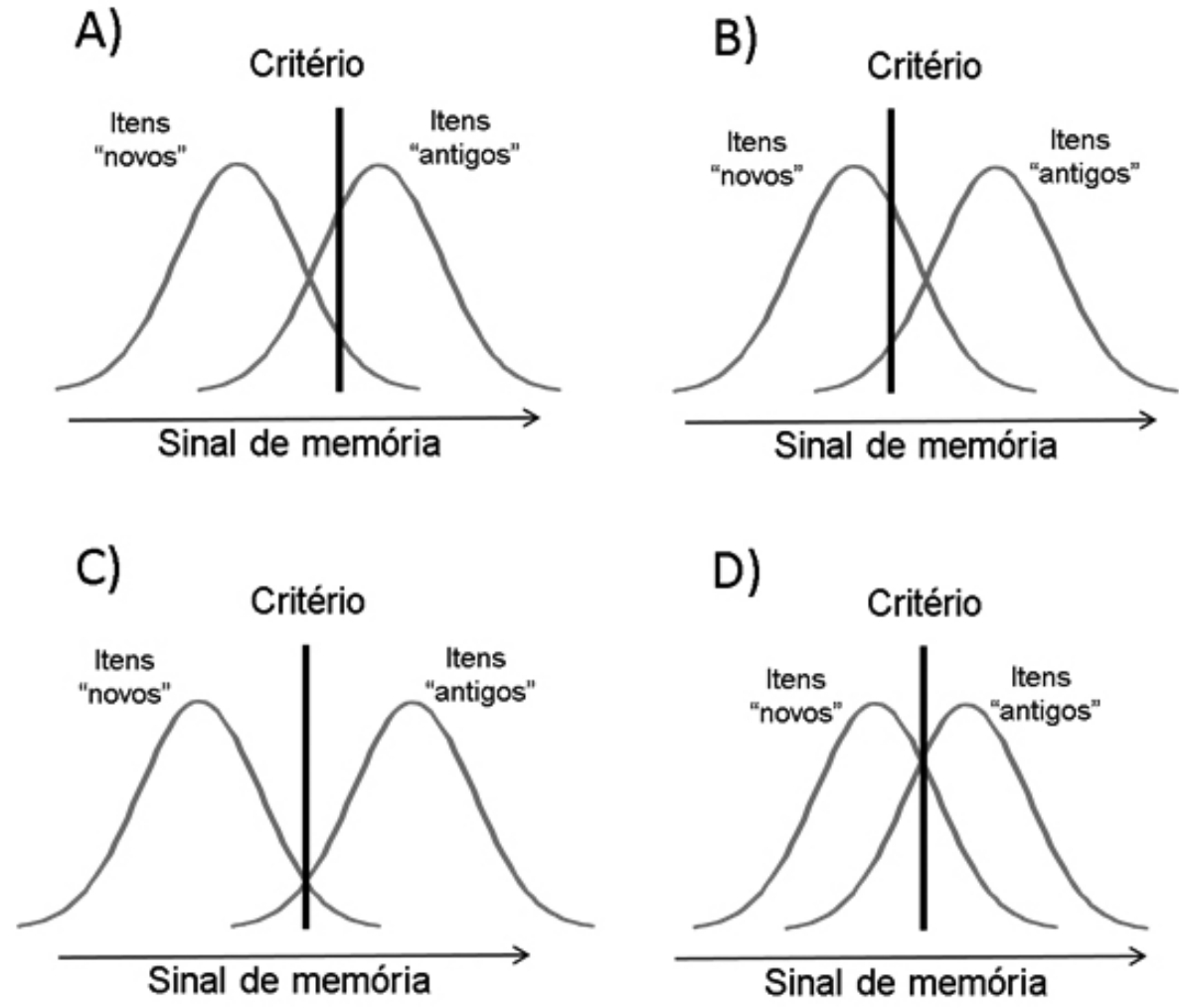

Figura 3. Critério e discriminabilidade de acordo com a teoria da detecção de sinal.

Figura 3. Pressupostos do modelo de detecção de sinal quanto a mudança em critério (A e B) e discriminabilidade (C e D). Na Figura 2A, um critério conservador é adotado, levando a maiores índices de rejeições corretas e a menores índices de acertos. Na Figura 2B, um critério mais liberal é adotado, o que leva a um aumento nos índices de acertos e a uma diminuição nos índices de rejeições corretas. Na Figura 2C o maior afastamento das curvas indica maior capacidade de discriminabilidade entre itens antigos e novos, enquanto na Figura $2 \mathrm{~B}$ a maior proximidade entre as curvas representa menor discriminabilidade entre itens antigos e novos. 
de discriminar entre essas duas classes de estímulo é menor.

Outra maneira importante de se visualizar e de se analisar dados dentro do escopo da teoria da Detecção de Sinal é utilizando curvas ROC (Receiver Operating Characteristics) (Luce, 1963). Para que curvas ROC sejam implementadas, entretanto, é necessário, que durante o teste de reconhecimento, níveis de confiança também sejam coletados. Usualmente, isso é feito pedindo-se aos participantes de pesquisa que estimem o quanto estão confiantes em cada uma de suas respostas dentro de uma escala de 1 a 6 . Assim, 1 pode significar confiança alta; 2 , confiança média e 3 , confiança baixa de que o estímulo é antigo, enquanto 4 pode significar confiança baixa; 5 , confiança média e 6 , confiança alta de que o estímulo é novo. Para que a curva ROC seja composta, as proporções para essas respostas devem ser apresentadas nela de forma cumulativa. Assim, como pode ser visto na Figura 4A, no eixo vertical, são apresentadas as proporções cumulativas para os acertos, enquanto, no eixo horizontal, as proporções cumulativas para alarmes falsos.

Dessa forma, o ponto mais abaixo e a esquerda da curva ROC (Figura 4A), consiste na proporção de acertos com alta confiança (eixo vertical) em função da proporção de alarmes falsos com alta confiança (eixo horizontal). O ponto em seguida representa a proporção de acertos com alta confiança somados à proporção de acertos com confiança média, em função da proporção de alarmes falsos com alta confiança somados aos alarmes falsos de confiança média. Os pontos conseguintes seguem a mesma lógica, sendo que o sexto ponto não é inserido por resultar em valor absoluto.
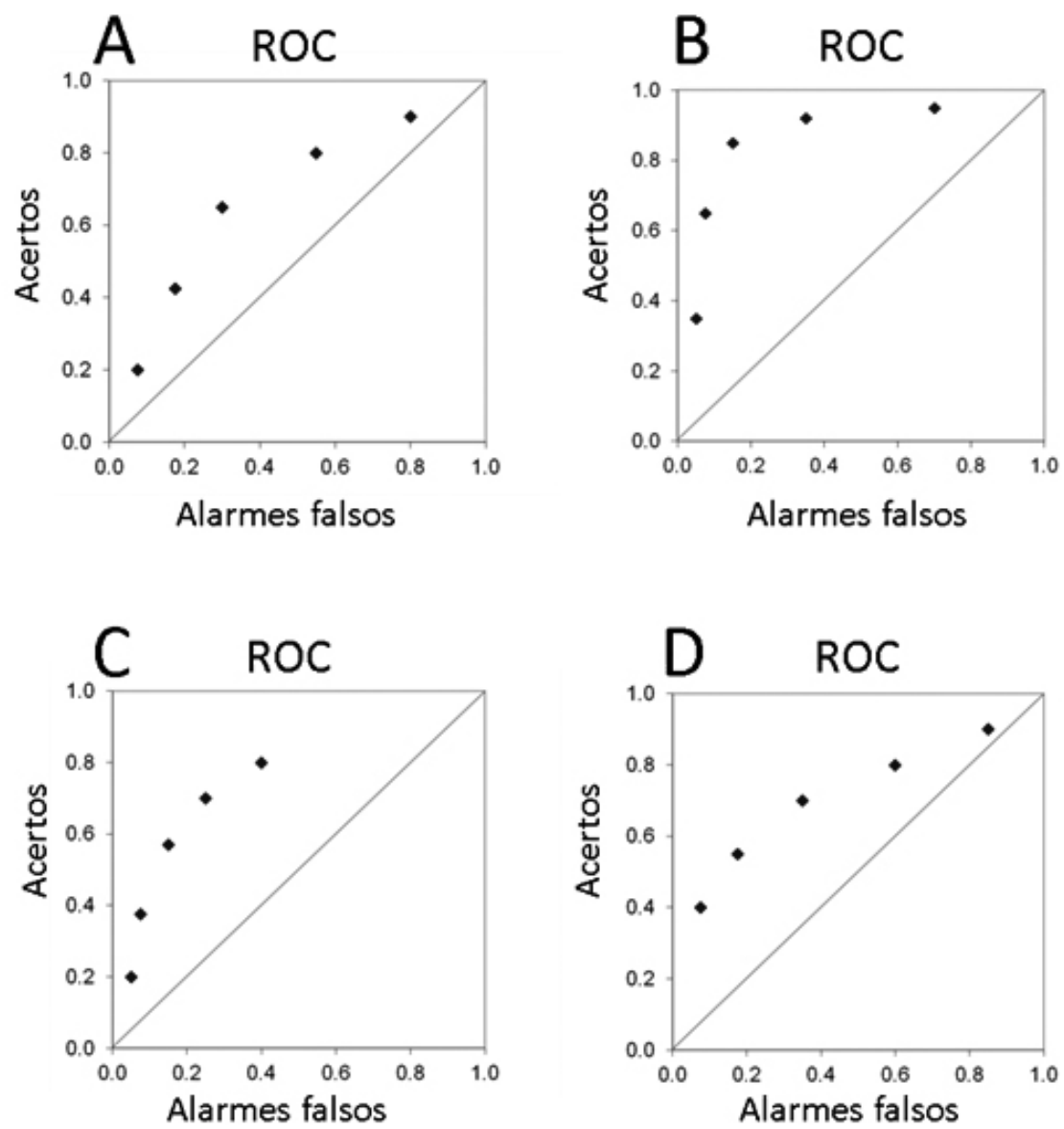

Figura 4. Curvas de ROC.

Figura 4. Curva ROC de acordo com a predição da teoria da detecção de sinal (A); curva ROC demonstrando uma discriminabilidade alta entre itens novos e antigos (B); curva ROC demonstrando a adoção de um critério conservador por sujeito hipotético $(\mathrm{C})$; curva ROC não prevista pela teoria da detecção de sinal (D). 
A capacidade de discriminar entre itens antigos e novos de cada indivíduo é representada na curva ROC por meio do posicionamento dos pontos da curva em direção ao canto superior esquerdo da figura (Egan, 1975), como pode ser visto na Figura 4B. Assim, um aumento na discriminabilidade significa um posicionamento dos pontos da curva de modo mais próximo ao ângulo superior esquerdo. Em contrapartida, quanto mais próximos os pontos da curva estiverem da linha diagonal, menor a discriminação feita pelo indivíduo. Quando os pontos da curva são localizados sobre a linha diagonal, por exemplo, o desempenho é considerado aleatório, significando que o indivíduo não foi capaz de discriminar entre antigo e novo, e estaria apresentando, nesse caso, a mesma proporção de "acertos" e "alarmes falsos" (Verde \& Rotello, 2007).

Critério, por outro lado, pode ser observado nas curvas ROC por meio do posicionamento dos pontos da curva ao longo da linha diagonal (Macmillan \& Creelman, 2005). Na Figura 4C, por exemplo, os pontos da curva estão mais distribuídos sobre o lado esquerdo da linha diagonal, significando que a quantidade de alarmes falsos foi relativamente baixa, indicando que o sujeito, nesse caso, teria adotado um critério mais conservador (i.e., evitando emitir alarmes falsos, ainda que isso diminua o número de acertos) (ver Figura 3A). Caso os pontos da curva estejam distribuídos predominantemente sobre o lado direito da curva diagonal, isso sugeriria que o sujeito estaria utilizando um critério mais liberal, isto é, estaria emitindo muitos "acertos", mas também muitos "alarmes falsos" (ver Figura 3B).

Um pressuposto importante da teoria da Detecção de Sinal é de que a distribuição dos pontos da curva deve ser simétrica em relação a linha diagonal, como demonstrado na Figura 4A (Luce, 1963; Macmillan \& Creelman, 2005). Assim, ainda que a curva se modifique de acordo com discriminabilidade e critério, isso deve ocorrer de maneira simétrica em relação ao eixo diagonal (i.e., os pontos do lado esquerdo e direito da curva devem possuir distâncias idênticas à linha diagonal quando não há diferença em critério). Em realidade, nem sempre é isso que ocorre. Como exemplificado na Figura 4D, inúmeros estudos de reconhecimento de memórias demonstram que a curva ROC se mostra assimétrica, com os pontos de maior confiança (pontos à esquerda da curva) mais distantes da curva diagonal em comparação com os pontos de menor confiança (pontos à direita). Essa assimetria significa que a quantidade de acertos reconhecidos com confiança alta é maior do que a quantidade de alarmes falsos reconhecidos com confiança alta. Esse padrão é comparativamente diminuído para os itens de confiança baixa (Yonelinas, Dobbins, Szymanski, Dhaliwal, \& King, 1996).

Essa anomalia encontrada nos resultados empíricos, para utilizar o termo proposto por Thomas Kuhn, foi exposta há aproximadamente duas décadas (Yonelinas, 1994) e desde então ela tem sido abordada por duas linhas de pensamento bastante divergentes. A primeira consiste em uma versão modificada da teoria da Detecção de Sinal (Mickes et al., 2007) e sugere que as distribuições do sinal de memória para itens antigos e novos apresentam variâncias diferentes durante o teste de reconhecimento (Figura5A). Isto é, a distribuição dos itens antigos possui uma variância aproximadamente $20 \%$ maior do que a distribuição dos itens novos. A segunda linha de pensamento que aborda a assimetria nas curvas ROC é a teoria do Processamento Duplo (Yonelinas \& Parks, 2007), a qual será discutida em maior detalhe na seção seguinte.

\section{Teoria do Processamento Duplo}

A teoria do Processamento Duplo propõe que o reconhecimento de memórias pode envolver dois processos distintos: familiaridade e recordação. O primeiro consiste em um sinal de memória como o proposto pela teoria da Detecção de Sinal. O segundo envolve a evocação de aspectos contextuais e mais detalhados do evento memorizado (Mandler, 1980; Jacoby, 1991; Yonelinas, 1994, 2002; Yonelinas \& Parks, 2007; Tulving, 1985, 2002; Yu, Johnson, \& Rugg, 2012). Assim, quando um indivíduo desempenha um teste de reconhecimento para uma lista de palavras, por exemplo, ele pode julgar cada palavra, baseando-se em uma sensação de familiaridade, assim como pode também fazer esse julgamento, baseando-se na evocação de aspectos específicos experienciados durante a codificação (Figura 5B), como por exemplo, o que o indivíduo pensou ou sentiu na hora que viu a palavra na fase de codificação.

Essa abordagem teórica foi especialmente apoiada pela assimetria encontrada nas curvas ROC (Yonelinas, 1994, Yonelinas et al. 1996). Uma vez que essa assimetria é causada por uma quantidade desproporcional de acertos reconhecidos com alta confiança relativo a alarmes falsos reconhecidos com alta confiança, pode-se supor que subjacente a esse efeito se encontra o processo de recordação. Isto é, quando itens eliciam o processo de recordação, eles são classificados com alta confiança. Assim, levando-se em conta que uma parcela significativa dos itens antigos elicia o processo de recordação, e de que a recordação é um processo 
A Detecção de Sinal
(variância diferente)

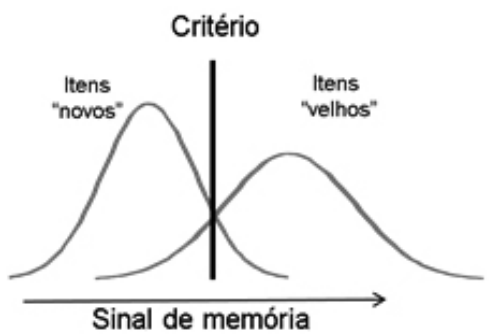

\section{B Processamento Duplo}

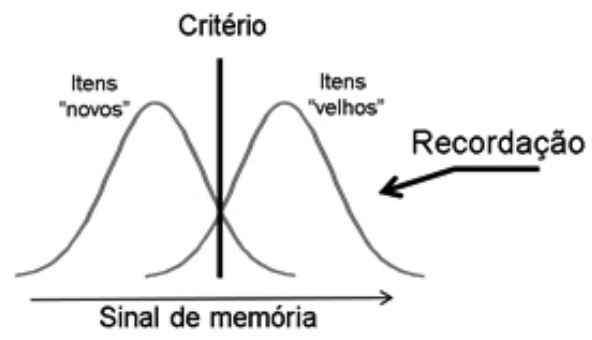

Figura 5. Modelo de detecção de sinal com variância diferente e modelo de processamento duplo.

Figura 5. Modelo de detecção de sinal com variância diferente para itens novos e antigos (A); e Modelo de processamento duplo (B).

virtualmente ausente em alarmes falsos (Yonelinas \& Parks, 2007), os itens que eliciam recordação acabam gerando a elevação do lado esquerdo da curva (Figura 4D) e, consequentemente, a assimetria encontrada nas curvas ROC.

Além das evidências provenientes das curvas ROC, a teoria do Processamento Duplo vem recebendo suporte de diversas abordagens científicas, como, por exemplo, da neurociência cognitiva. Estudos utilizando Potenciais Relacionados ao Evento (PRE), por exemplo, demonstram que os processos de familiaridade e recordação eliciam ativações eletrofisiológicas qualitativamente dissociáveis durante testes de reconhecimento (Wang, Chastelaine, Minton, \& Rugg, 2012; Jaeger, Johnson, Corona, \& Rugg, 2009; Jaeger \& Parente, 2008; Rugg \& Curran, 2007; Vilberg, Moosavi, \& Rugg, 2006), sugerindo de modo contundente que familiaridade e recordação consistem em processos distintos.

A utilização de técnicas de imageamento cerebral, como a ressonância magnética funcional (RMF), também tem gerado evidências favoráveis à teoria do Processamento Duplo. Um debate vigente gerado por resultados dessa técnica consiste na identificação de quais regiões cerebrais suportam o processo de familiaridade, e quais regiões suportam o processo de recordação. Assim, alguns estudos sugerem que o giro angular, uma região que faz parte do lobo parietal, assim como o hipocampo, tem um papel crucial no processo de recordação (Vilberg \& Rugg, 2012), enquanto regiões mais superiores do lobo parietal e regiões corticais adjacentes ao hipocampo estariam envolvidas no processamento da familiaridade (Yonelinas, Otten, Shaw, \& Rugg, 2005).
Inúmeros experimentos comportamentais também têm gerado dados favoráveis à teoria do Processamento Duplo (ver Yonelinas e Parks, 2007 para uma revisão). Um estudo recente utilizou um paradigma experimental no qual pistas influenciavam o julgamento de memórias durante a fase de teste (Jaeger, Cox, \& Dobbins, 2012). Mais especificamente, os itens na fase de teste eram precedidos por pistas que indicavam de maneira probabilística se eles haviam ou não haviam sido apresentados na fase de codificação. Assim, os itens eram precedidos por pistas como "provavelmente antigo", denotando que aquele item específico provavelmente havia sido apresentado na fase de codificação, ou "provavelmente novo", denotando que aquele item específico provavelmente não havia sido apresentado durante a fase de codificação. Essas pistas eram 75\% do tempo corretas (válidas) e $25 \%$ do tempo incorretas (inválidas). Além disso, quando os participantes faziam os julgamentos de memória, eles também realizavam uma estimativa da confiança sobre suas respostas.

Foi demonstrado por meio deste estudo que o reconhecimento de itens antigos e o reconhecimento de itens novos não poderiam ser igualmente explicados por um sinal contínuo de memória, o que é previsto pelo modelo de detecção de sinal (Macmillan \& Creelman, 2005). Isto é, esse paradigma demonstrou que as pistas influenciam de maneira diferenciada o julgamento de memória de itens antigos e o julgamento de itens novos. Os dados demonstraram que enquanto índices de desempenho e confiança foram igualmente influenciados pelas pistas no caso dos itens novos (desempenho e confiança aumentavam com pistas válidas e diminuíam com pistas inválidas), as pistas 
influenciaram de maneira diferente desempenho e confiança quando itens antigos estavam sendo julgados, ou seja, enquanto o desempenho era prejudicado por pistas inválidas e melhorado por pistas válidas, o índice de confiança era o mesmo para as pistas válidas e inválidas (Jaeger et al., 2012). Uma vez que a teoria da Detecção de Sinal assume uma relação simétrica entre desempenho e confiança (Stretch \& Wixted, 1998), esses dados se apresentam como uma refutação dos pressupostos dessa teoria.

Em suma, estudos utilizando diversas abordagens comportamentais e neurocientíficas sugerem que um simples sinal contínuo de intensidade de memória não é suficiente para explicar o reconhecimento de memórias e apoiam a teoria de que o reconhecimento de memórias envolve dois processos: familiaridade e recordação (Mandler, 1980; Jacoby, 1991; Yonelinas, 1994). A familiaridade consiste em um sinal gradativo ou contínuo, como inicialmente referido pela teoria da Detecção de Sinal, enquanto a recordação consiste em uma recuperação vívida e consciente do episódio vivenciado, assim como, o contexto envolvido em cada episódio.

\section{Discussão Crítica e Considerações Finais}

Uma parcela importante da literatura atual sobre reconhecimento de memórias episódicas se dedica a dar suporte para um dos dois modelos teóricos apresentados acima (teoria da Detecção de Sinal ou teoria do Processamento Duplo). Desde a descoberta da assimetria presente nas curvas ROC em tarefas de reconhecimento de memórias, o modelo de detecção de sinal clássico, como proposto por Green e Swets durante os anos sessenta (Green \& Swets, 1966), teve sua validade como um modelo de reconhecimento de memórias bastante questionada. Um resultado disso é que, atualmente, adeptos da teoria da Detecção de Sinal para o reconhecimento de memórias, de forma geral, adotam o modelo proposto por Mickes, Wixted e Wais (2007), segundo o qual há uma diferença entre a variabilidade do sinal de memória para itens antigos e novos.

Talvez o principal motivo pelo qual a teoria da Detecção de Sinal não tenha sido completamente abandonada durante os últimos anos, tenha sido a sua grande flexibilidade em dar conta dos dados empíricos. A comparação entre os modelos da detecção de sinal e de processamento duplo, geralmente ocorrem por meio do que é chamado de model fitting, o que conceitualmente consiste na avaliação estatística do quanto os dados empíricos se aproximam dos dados previstos pelo modelo, levando em conta os parâmetros de cada modelo. Embora uma discussão dessa metodologia exigiria uma introdução extensa à utilização de modelos em Psicologia Cognitiva, o que não é o objetivo deste manuscrito, pode-se brevemente afirmar que tanto o modelo do processamento duplo, como o da detecção de sinal geralmente mostram boa proximidade com os dados empíricos por meio dessas análises. Esse método, entretanto, é considerado uma abordagem post-hoc, ou seja, implementada a posteriori e sujeita a não ser efetiva quando os modelos apresentam uma quantidade elevada de parâmetros variáveis, como no caso dos dois modelos aqui discutidos (Pitt, Kim, \& Myung, 2003).

Uma abordagem alternativa é hipotetizar previamente como os dados empíricos deverão se mostrar a partir dos pressupostos de cada modelo e, em seguida, comparar dados empíricos com essas previsões. Essa abordagem foi utilizada por Jaeger, Cox e Dobbins (2012), no experimento descrito na seção anterior. Esses autores simularam previamente como as pessoas deveriam responder à tarefa, caso o modelo de detecção de sinal ou processamento duplo fossem "verdadeiros" (ou seja, caso eles realmente descrevessem como as pessoas reconhecem memórias). Posteriormente, os dados simulados foram comparados com os dados empíricos emitidos pelos participantes de pesquisa. Essa comparação foi extremamente favorável ao modelo de processamento duplo em comparação com o modelo de detecção de sinal.

Assim como as análises de dados comportamentais discutidas acima, técnicas neurocientíficas são cada vez mais utilizadas no debate entre modelo de processamento duplo e detecção de sinal. Quando esses achados são levados em conta, o modelo de processamento duplo é bastante favorecido, pois a demonstração de que há dois processos que envolvem substratos neurais independentes, contradiz o pressuposto básico da teoria da Detecção de Sinal aplicada ao reconhecimento de memórias, que é o de que um processo simples de familiaridade é suficiente para o reconhecimento.

Uma questão importante, mas pouco debatida, é o quanto as memórias evocadas em testes de reconhecimento realmente configuram memórias episódicas, segundo o conceito proposto por Endel Tulving (Tulving, 2002). Como mencionado na introdução, a memória episódica envolve consciência autonoética, o que se caracteriza por uma sensação subjetiva de que o evento recordado foi vivenciado em primeira pessoa e se refere a um evento real previamente vivenciado pelo indivíduo (Tulving \& Thomson, 1973). 
É necessário consciência autonoética para possuir uma sensação de familiaridade? Quando se tem uma sensação de familiaridade em relação a determinado estímulo, isso configura memória episódica? Essas são questões teóricas ainda não resolvidas, ainda que o que parece emergir dessas questões é que, enquanto o processo de recordação, como definido pelo modelo de processamento duplo (Yonelinas, 2002) parece envolver processos de memória episódica, isso não parece ocorrer para o processo de familiaridade. Assim, o componente episódico do processo de reconhecimento seria o próprio processo de recordação, enquanto é discutível o quanto o processo de familiaridade envolve aspectos episódicos propriamente ditos, pois ele envolve apenas "o que" foi memorizado, e não "quando" e "onde", que são aspectos inerentes à evocação episódica (Tulving, 2002).

Em suma, embora a teoria da Detecção de Sinal seja uma teoria elegante e que tenha gerado uma série de medidas e índices úteis para o estudo da memória, o contraste entre os modelos de processamento único e duplo desenvolvido e discutido no presente artigo, sugere que a combinação de dois processos cognitivos é necessária para produzir o fenômeno do reconhecimento de memórias: os processos de familiaridade e de recordação. Futuras pesquisas ainda serão importantes para definir de forma mais precisa o papel de cada um desses processos para que o reconhecimento de memórias seja possível.

\section{Referências}

Banks, W. P. (1970). Signal detection theory and human memory. Psychological Bulletin, 74, 81-99.

Egan, J. P. (1975). Signal detection theory and ROC analysis. New York: Academic press.

Glanzer, M., \& Cunitz, A. (1966). Two storage mechanisms in free recall. Journal of Verbal Learning and Verbal Behavior, 5, 351-360.

Green, D.M., Swets J.A. (1966) Signal detection theory and psychophysics. New York: Wiley.

Greene, R. L. (1986). Word stem as cues in recall and completion tasks. The Quarterly Journal of Expeirmental Psychology, 38, 663-673.

Jacoby, L. L. (1991). A process dissociation framework: Separating automatic from intentional uses of memory. Journal of Memory and Language, 30, 513-541.
Jaeger, A., Cox, J. C., \& Dobbins, I. G. (2012). Recognition confidence under violated and confirmed memory expectations. Journal of Experimental Psychology: General, 141, 282-301.

Jaeger, A., Johnson, J. D., Corona, M., \& Rugg, M. D. (2009). ERP correlates of the incidental retrieval of emotional information: Effects of study-test delay. Brain Research, 1269, 105-113.

Jaeger A., \& Parente, M. A. M. P. (2008). Event-related potentials and the study of memory retrieval. Dementia \& Neuropsychology, 2, 248-255.

Lockhart, R. S., \& Murdock, B, B. (1970). Memory and the theory of signal detection. Psychological Bulletin, 74, 100-109.

Luce, R. D. (1963). Detection and Recognition. Em R. D. Luce, R. R. Bush, \& E. Galanter (Eds.), Handbook of mathematical psychology (pp. 103-189). New York: Wiley.

Macmillan, N. A., \& Creelman, C. D. (2005). Detection theory: A user's guide (2nd ed.). New York: Cambridge University Press.

Mandler, G. (1980). Recognizing: The judgment of previous occurrence. Psychological Review, 87, 252-271.

Mickes, L., Hwe, V., Wais, P. E., \& Wixted, J. T. (2011). Strong memories are hard to scale. Journal of Experimental Psychology: General, 140, 239-257.

Mickes, L., Wixted, J. T., \& Wais, P. E. (2007). A direct test of the unequal-variance signal detection model of recognition memory. Psychonomic Bulletin \& Review, 14, 858-865.

Pitt, M. A., Kim, W., \& Myung, I. J. (2003). Flexibility versus generalizability in model selection. Psychonomic Bulletin \& Review, 10, 29-44.

Rugg, M. D., \& Curran, T. (2007). Event-related potentials and recognition memory. Trends in Cognitive Sciences, 11, 251-257.

Stretch, V., \& Wixted, J. T. (1998). Decision rules for recognition memory confidence judgments. Journal of Experimental Psychology: Learning, Memory, and Cognition, 24, 1397-1410.

Tulving, E. \& Thomson, D. M. (1973). Encoding specificity and retrieval processes in episodic memory. Psycbological Review, 80, 352-373. 
Tulving, E. (1983). Elements of episodic memory. Oxford: Clarendon Press.

Tulving, E. (1985). Memory and consciousness. Canadian Psychology, 26, 1-12.

Tulving, E. (2002). Episodic memory: From mind to brain. Annual Review of Psychology, 53, 1-25.

Verde, M. F., \& Rotello, C. M. (2007). Memory strength and the decision process in recognition memory. Memory \& Cognition, 35, 254-262.

Vilberg, K. L., \& Rugg, M. D. (2012). The neural correlates of recollection: Transient versus sustained fMRI effects. The Journal of Neuroscience, 32, 15679-15687.

Vilberg, K. L., \& Moosavi, R. F., Rugg, M. D. (2006). The relationship between electrophysiological correlates of recollection and amount of information retrieved. Brain Research, 1122, 161-170.

Wang, T. H., de Chastelaine, M., Minton, B., \& Rugg, M. D. (2012). Effects of age on the neural correlates of familiarity as indexed by ERPs. Journal of Cognitive Neuroscience, 24, 1055-1068.

Yonelinas, A. P. (1994). Receiver-operating characteristics in recognition memory: Evidence for a dual-process model. Journal of Experimental
Psychology: Learning, Memory, and Cognition, 20, 1341-1354.

Yonelinas, A. P. (2002). The nature of recollection and familiarity: A review of 30 years of research. Journal of Memory and Language, 46, 441-517.

Yonelinas, A. P., Otten, L. J., Shaw, K. N., \& Rugg, M. D. (2005). Separating the brain regions involved in recollection and familiarity in recognition memory. The Journal of Neuroscience, 25, 3002-3008.

Yonelinas, A. P., Parks, C. M. (2007). Receiver operating characteristics (ROCs) in recognition memory: A review. Psychological Bulletin, 133, 800-832.

Yonelinas, A. P., Dobbins, I. G., Szymanski, M. D., Dhaliwal, H. S., \& King. L. (1996). Signal-detection, threshold, and dual-process models of recognition memory: ROCs and conscious recollection. Consciousness \& Cognition, 5, 418-441.

Yu, S. S., Johnson, J. D., \& Rugg, M. D. (2012). Hippocampal activity during recognition memory co-varies with the accuracy and confidence of source memory judgments. Hippocampus, 22, 1429-1437.

Recebido 17/04/2015

Reformulado 05/08/2015

Aceito 01/09/2015

Sobre o autor:

Antônio Jaeger é psicólogo, doutor em Psicologia pela UFRGS, com pós-doutorado pela Washington University in St Louis, USP e PUCRS, professor adjunto do Departamento de Psicologia da UFMG e professor da pós-graduação em Neurociências e da Pós-graduação em Psicologia da UFMG.

Contato com o autor:

Antônio Jaeger, Departamento de Psicologia, FAFICH, Universidade Federal de Minas Gerais

Av. Antônio Carlos, 6627

CEP: 31270-901

Belo Horizonte-MG, Brasil

E-mail: antonio.jaeger@gmail.com

Fones: (31) 9527-8515, (31) 3409-6279. 